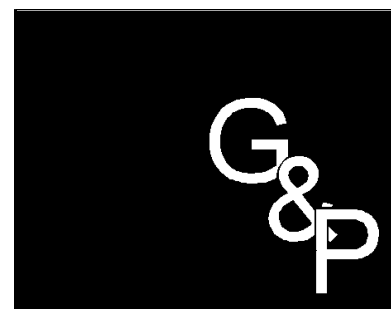

\section{GESTÃO}

$\&$

\section{PRODUÇÃO}

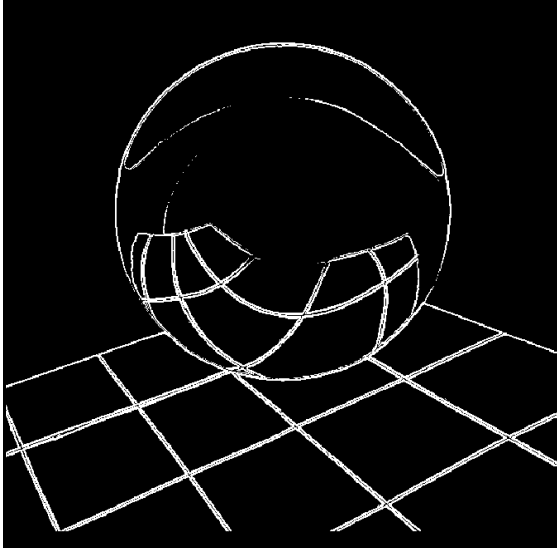

v.4, n.3, p. 305-320, dez. 1997

\title{
UMA METODOLOGIA BAYESIANA PARA ESTUDOS DE CONFIABILIDADE NA FASE DE PROJETO: APLICAÇÃO EM UM PRODUTO ELETRÔNICO
}

\author{
Ruth Myriam Ramírez Pongo \\ Escola Politécnica da USP \\ Depto. de Engenharia de Produção \\ Pedro Rodrigues Bueno Neto \\ Escola Politécnica da USP \\ Depto. de Engenharia de Produção
}

\section{Resumo}

Hoje em dia as indústrias têm de colocar seus produtos em um mercado altamente competitivo, e as pressões, visando eficiência do programa de testes e do ciclo de desenvolvimento de um produto, são cada vez maiores. Neste contexto, os métodos estatísticos clássicos (baseados na teoria de amostragem), usados para analisar a confiabilidade de um produto, mostram-se cada vez menos eficientes, enquanto que os métodos bayesianos são vistos favoravelmente e seu uso começa a ser uma alternativa necessária.

Para avaliar o desempenho de um item, que possui uma taxa de falhas muito baixa, as técnicas estatísticas clássicas exigem tamanhos grandes de amostra e tempo de teste longo, principalmente quando a tecnologia do produto limita o fator de aceleração, como é o caso dos produtos eletrônicos, por exemplo.

A metodologia, proposta neste artigo, combina resultados de testes, que são realizados rotineiramente durante o ciclo de desenvolvimento, com informação adicional relevante e útil ao estabelecimento da confiabilidade do equipamento.

Com o objetivo de ilustrar a metodologia, esta é aplicada em um equipamento eletrônico, cuja confiabilidade foi estabelecida, durante sua fase de projeto, a partir das confiabilidades de seus componentes, utilizando dados de teste por atributo e incorporando o julgamento da equipe de desenvolvimento do produto.

Palavras-chave: confiabilidade, estatistica bayesiana, qualidade, taxa de falla, opinião de especialista. 
1. Introdução

$\mathrm{A}$ vanços rápidos da tecnologia, desenvolvimento de produtos altamente sofisticados, competição intensamente global, juntamente com a crescente expectativa do consumidor têm-se combinado para impor novas pressões aos fabricantes para produzir produtos de alta qualidade. Os clientes esperam comprar produtos que sejam confiáveis e seguros, sendo que sua satisfação é conseguida durante a vida útil do produto, e não apenas na ocasião da compra. A confiabilidade reflete a probabilidade de bom funcionamento de um produto, ou de ele não falhar, em um determinado período de tempo. Dentre as medidas mais comuns da confiabilidade estão o tempo médio para a primeira falha, o tempo médio entre falhas e a taxa de falhas por unidade de tempo.

Historicamente, as previsões ou medidas indiretas de confiabilidade baseadas em testes de laboratório, têm sido usadas de maneira eficiente (apesar de serem potencialmente inexatas) para verificar se a linha de fabricação de um item atende à especificação de taxas de falhas. Taxas de falhas, devido a defeitos aleatórios, têm sido estimadas com sucesso usando dados de testes de vida acelerados do item em estudo, os quais são convertidos a condições normais de operação uma vez que os mecanismos de falha sejam entendidos.

Na fabricação de produtos eletrônicos, o teste de vida acelerado e a análise de falha post-mortem, como metodologia de confiabilidade, está sendo cada vez menos eficiente, na medida que os produtos têm especificações menores para as taxas de falhas, e na medida que se tornam mais complexos, com tamanhos menores e maior sofisticação de operacionalidade.

Por exemplo, embora parecesse que as leis da física limitassem a demanda de melhoria contínua do desempenho e densidade dos chips, a realidade indica que esta tendência continuará, e certamente ela causará um impacto na confiabilidade dos componentes eletrônicos. As projeções indicam que, na mudança do século, os chips de microcomputadores terão 100 milhões de transistores e as taxas de falhas serão menores que 10 FIT (Failure In Time, 1 falha em um milhão de componentes operando por 1000 horas). Isto significa que as técnicas de medição de confiabilidade terão sérias limitações para lidar com taxas de falhas de 10 FIT dos circuitos impressos.

Em oposição à necessidade de mais teste para alcançar alta qualidade e confiabilidade, existe, hoje em dia, uma pressão extrema para reduzir a extensão de tempo entre a concepção do produto e sua produção. Esta pressão reduz ainda mais o tempo disponível para testar a confiabilidade em todos os estágios do processo de desenvolvimento do produto. Isto cria novos desafios para o projeto do produto e processo de produção e para o planejamento e análise dos resultados dos estudos de confiabilidade, a fim de reduzir o risco de não detectar problemas potenciais de confiabilidade.

Combinar em um plano de teste, de maneira ótima, o número de unidades de ensaio, tempo de teste, nível de aceleração e nível de confiança para se obter inferências de boa qualidade sobre a confiabilidade de um item em estudo, sob os princípios da teoria de amostragem, está se tornando um problema cada vez mais crítico. Se a esta situação adicionarmos a característica do produto observado ser altamente confiável, o que significa que os dados de falha são escassos, então fica praticamente inviável achar um ponto de equilíbrio entre o uso de metodologias científicas e o desafio da empresa concorrer de maneira competitiva em um mercado altamente agressivo. 
Diante destes fatos, surgem, do meio de engenharia de desenvolvimento, as seguintes questões: 1) $\mathrm{O}$ ensaio proposto atende ao objetivo do teste de demonstração de confiabilidade? 2) Quanta confiança, e/ou melhoria, o ensaio proposto acrescentará à qualidade do produto? 3) Se a fabricação dos produtos exige a prévia qualificação de componentes, projeto e processo, este ambiente de manufatura não favorece, de alguma forma, a certeza nas afirmações feitas sobre a confiabilidade do produto?

Os projetos de engenharia são processos evolucionários, e isto proporciona ao desenvolvedor um conhecimento técnico e experiência que o habilita a possuir julgamentos subjetivos de especialista ("expert opinion"), além do que, informação valiosa sobre o produto em desenvolvimento pode ser obtida dos ensaios realizados em protótipos. A pergunta é: como aproveitar e formalizar a utilização desta informação nos cálculos para estimar a confiabilidade de um produto?

Nossa proposta é a de fornecer uma metodologia rápida e econômica para quantificar toda informação (subjetiva e objetiva) relevante ao estudo, baseada em métodos de inferência bayesiana. Alguns trabalhos de aplicação em problemas práticos podem ser citados, por exemplo os de: MASTRAN (1968), COLE (1975), HART (1990), MARTZ \& WALLER (1990), entre outros. Os benefícios desta metodologia estão alinhados com o desafio atual que as empresas devem enfrentar: desenvolvimento rápido de produtos altamente confiáveis com custos otimizados.

\section{Métodos Bayesianos em Confiabilidade}

abordagem bayesiana em inferên-
cia estatística propõe-se a combinar
dados obtidos de observações com avaliações ou julgamentos subjetivos. Em muitos problemas de confiabilidade, a utilização dos métodos bayesianos apresenta-se não como uma opção e sim como uma necessidade. No estudo de confiabilidade em novos protótipos, principalmente quando estes são disponíveis em número extremamente reduzido - às vezes um ou dois itens - a inferência estatística clássica não dá respostas adequadas, pois não permite o uso da experiência anterior com modelos similares, nem a opinião dos especialistas da área. Surge assim a teoria bayesiana como a ferramenta indicada para o aproveitamento de toda a informação disponível, seja ela objetiva, fornecida pelos resultados de testes, ou subjetiva, ditada pela experiência.

Por meio dos métodos bayesianos é possível incorporar, à análise estatística, convicções, conhecimento prévio e opiniões. Para conseguir isto vale-se dos argumentos sobre probabilidades condicionais. Nesta formulação, para uma variável aleatória $X$, com função densidade de probabilidade (f.d.p.) $f(x)$, supõe-se que esta densidade depende de um parâmetro desconhecido $\theta$. Na teoria estatística clássica o parâmetro é considerado uma constante fixa. Na teoria bayesiana se introduz a convicção pessoal nos possíveis valores de $\theta$, considerando-a como uma variável aleatória $\Theta$, com uma densidade $h(\theta)$, denominada distribuição $\boldsymbol{a}$ priori de $\Theta$. Por esta densidade representa-se quantitativamente nosso grau de convicção, ou a força de nossa opinião, a favor de certos conjuntos de valores de $\Theta$. Não estamos dizendo que $\theta$ não seja uma constante, mas apenas que, pelo fato de não sabermos seu valor exato mas termos uma razoável idéia de quais são os possíveis valores desta constante, com certezas diferentes para estes valores, modelamos este conhecimento por uma distribuição de probabilidades.

A introdução da distribuição a priori tem duas conseqüências. Primeiro, $f_{\Theta}(\theta)$ con- 
terá freqüentemente informação sobre $o$ valor de $\theta$, e supondo que esta informação seja correta, a inferência sobre $\theta$ será refinada. Segundo, o fato de que $\Theta$ seja uma variável aleatória esclarece grandemente os métodos de análise a serem utilizados.

$\mathrm{Na}$ prática, poderá acontecer duas situações de interesse: a) obtenção de informação sobre o parâmetro $\Theta$ baseada na observação de variáveis aleatórias relacionadas; e b) obtenção de informação sobre uma observação futura, $X^{\dagger}$, que depende de $\theta$. Na primeira situação, quando $X=x$ é observado, será necessário calcular a f.d.p condicional de $\Theta$ dado $X=x$; esta é a denominada distribuição $\boldsymbol{a}$ posteriori. $\mathrm{Na}$ segunda situação, será possível obter a distribuição condicional do valor $X^{\dagger}$ dado os dados da distribuição a posteriori de $\Theta$.

O mecanismo que combina a distribuição $a$ priori e os dados para formar a distribuição $a$ posteriori é o conhecido Teorema de Bayes, um resultado simples da teoria de probabilidades dado por Thomas Bayes em 1763.

Assim, se $X_{1}, X_{2}, \ldots, X_{n}$ constituem uma amostra aleatória, obtida da densidade $f(x)$, poder-se-á construir uma estatística $y=y\left(x_{1}, x_{2}, \ldots \ldots, x_{n}\right)$ como função dos valores observados na amostra. Para um valor $\theta$ dado, tem-se $f(y / \theta)$ que indica a densidade condicional de $y$ para $\theta$ dado. Pode-se também calcular a densidade conjunta de $y$ e $\Theta$ por meio da expressão:

$$
f_{Y, \Theta}(y, \theta)=h_{\Theta}(\theta) f_{Y / \Theta}(y / \theta)
$$

E se $\theta$ for contínua, a distribuição marginal de y será,

$$
f_{Y}(y)=\int h_{\Theta}(\theta) f_{Y / \Theta}(y / \theta) d \theta
$$

Logo, pelo Teorema de Bayes, a densidade condicional de $\theta$, dado $y$, será

$$
\begin{gathered}
h_{\Theta / Y}(\theta / y)=\frac{h_{\Theta}(\theta) f_{Y / \Theta}(y / \theta)}{f_{Y}(y)}, \\
f_{Y}(y)>0
\end{gathered}
$$

ou

$$
h_{\Theta / Y}(\theta / y)=\frac{h_{\Theta}(\theta) f_{Y / \Theta}(y / \theta)}{\int h_{\Theta}(\theta) f_{Y / \Theta}(y / \theta) d \theta}
$$

ou

$$
h_{\Theta / Y}(\theta / y) \propto f_{Y / \Theta}(y / \theta) h_{\Theta}(\theta)
$$

As equações 2.3 a 2.5 correspondem à expressão da distribuição a posteriori de $\Theta$, isto é, após a obtenção dos resultados de ensaios.

\section{Aplicação no Concentrador FDDI IBM 8240}

\subsection{Descrição do Produto}

$\mathrm{O}$

concentrador 8240 foi desenvolvido com base na arquitetura FDDI (Fiber Distributed Data Interface) e tem como função principal a de interconectar estações de trabalho e periféricos por meio de fibra óptica.

Como principal característica tem-se que sua velocidade de transmissão é de 100 Mbits por segundo; a possibilidade de conexão por fibra óptica ou cobre, a facilidade de instalação e o fato de acompanhar o padrão da indústria definido pelo ANSI (American National Standard Institute) para o FDDI são outras vantagens para os usuários.

Fisicamente, o IBM 8240 é constituído por uma estrutura metálica, uma fonte de alimentação, um circuito impresso mãe, um módulo de controle do concentrador, um módulo de conexão à rede e até seis módulos de ligação ao dispositivo. $\mathrm{O}$ projeto foi inteiramente desenvolvido e fabricado no Brasil no ano de 1991. 


\subsection{Ambiente de Desenvolvimento}

O desenvolvimento de um produto novo deve atender aos requisitos de um programa formal, que toda empresa especifica para garantir a qualidade dos seus produtos que serão lançados no mercado. A tabela abaixo relaciona as atividades de teste às diferentes fases de desenvolvimento de um produto, que por necessidade da aplicação usou-se a terminologia padrão IBM.

Tabela 3.1. Descrição dos testes realizados durante o desenvolvimento do produto.

\begin{tabular}{|l|l|}
\hline TESTES & DESCRIÇÃO \\
\hline EVT - Engineering Verification Test & $\begin{array}{l}\text { Verifica se os protótipos atendem à especificação funcional e } \\
\text { aos objetivos de desempenho do produto, como uma } \\
\text { preparação ao DVT. }\end{array}$ \\
\hline DVT - Design Verification Test & $\begin{array}{l}\text { Verifica se o projeto de hardware está completo, se atende à } \\
\text { especificação funcional, se é fabricável e se é adequado para } \\
\text { receber manutenção, em amostras da linha piloto. }\end{array}$ \\
\hline Guardband & $\begin{array}{l}\text { Teste conduzido para medir a robustez do projeto, que } \\
\text { consiste em levá-lo até a falha, fora da especificação, pela } \\
\text { variação de parâmetros ambientais e de operação. }\end{array}$ \\
\hline Usability & $\begin{array}{l}\text { O teste de "Usability" simula o ambiente do cliente em todas } \\
\text { suas fases, desde a desembalagem até o funcionamento do } \\
\text { produto. }\end{array}$ \\
\hline FVT - Functional Verification Test & $\begin{array}{l}\text { Verifica se o produto atende todos os requisitos definidos na } \\
\text { especificação funcional. }\end{array}$ \\
\hline SVT - System Verification Test & Verifica o desempenho do produto na rede. \\
\hline $\begin{array}{l}\text { MVT - Manufacturing Verification } \\
\text { Test }\end{array}$ & $\begin{array}{l}\text { Verifica se a produção em volume não impacta a qualidade do } \\
\text { produto. As amostras provêm da linha de produção final. }\end{array}$ \\
\hline
\end{tabular}

O EVT, DVT e MVT são testes direcionados a hardware (estrutura física do produto), microcódigo e fabricação, enquanto que as outras atividades são testes direcionados principalmente a microcódigo. Para tal fim, foram desenvolvidos uma série de softwares ("Test Cases"), focando a integridade do protocolo de comunicação, a simulação da operação no cliente, e a promoção e monitoramento do stress do hardware.

\subsection{Suposições do Modelo Proposto para o Estudo de Confiabilidade}

As afirmações subjetivas sobre a confiabilidade do concentrador são extraídas sob a suposição de que a vida útil deste produto é bem representada pela distribuição exponencial. A confiabilidade será revisada, partindo deste conhecimento, com os dados de testes obtidos durante seu desenvolvimento. Este estudo considera os seguintes itens:

- A análise está focada na confiabilidade do hardware;
- Taxa de falhas constante após o processo de burn-in (depuração);

- Configuração em série do produto;

- Uso de dados a priori e de teste de componentes;

- Baseado no design do produto, considera-se que as partes (ou componentes) falham de maneira independente;

- Fixado o tempo de teste, a confiabilidade é considerada como a probabilidade de sobrevivência, constante em cada ensaio; 
- A confiabilidade do produto será composta a partir das confiabilidades das partes;
- As estimativas poderão ser atualizadas toda vez que forem disponíveis dados amostrais de um novo hardware.

\subsection{Metodologia}

Estimação da confiabilidade, combinando dados de teste, com informação adicional, relevante ao conhecimento do produto, prévia aos ensaios. Este problema de inferência estatística é tratado aplicando a abordagem bayesiana.

\subsubsection{Formulação do Conhecimento, da Confiabilidade de Componente, Prévio às Atividades de Teste (Distribuição a Priori)}

Se, ao processar a operação de um componente, for observado seu sucesso ou falha, então se dirá que são disponíveis dados por atributo. Neste caso, a distribuição de amostragem é o modelo Binomial, com parâmetro $R_{i}$, onde $R_{i}=P\left(X_{i}=1\right)$ e o evento $X_{i}=1$ representa a operação satisfatória do i-ésimo componente.
De acordo com a abordagem bayesiana, o conhecimento prévio sobre a confiabilidade do componente, $R_{i}$, pode ser descrito mediante um modelo probabilístico. Para tal fim escolhe-se a distribuição Beta (7), uma família de distribuições a priori conjugada, i.e., uma família em que a distribuição a priori é tal que a distribuição a posteriori pertence à mesma família. Sua f.d.p. é dada por:

$$
h_{i}\left(R_{i}\right)=\frac{\Gamma\left(n_{\mathrm{i} 0}+2\right)}{\Gamma\left(s_{\mathrm{i} 0}+1\right) \Gamma\left(n_{\mathrm{i} 0}-s_{i 0}+1\right)} R_{i}^{s_{\mathrm{i} 0}}\left(1-R_{i}\right)^{n_{\mathrm{i} 0}-s_{\mathrm{i} 0}}
$$

Isto é:

$$
R_{i} \sim \beta\left(\mathrm{s}_{\mathrm{i} 0}+1, n_{\mathrm{i} 0}-s_{\mathrm{i} 0}+1\right)
$$

$s_{\mathrm{i} 0}$ é considerado como o número total de unidades que tiveram sucesso em um pseudoteste de $n_{\mathrm{i} 0}$ unidades.

A distribuição é especificada mediante a determinação de seus parâmetros. Estes foram determinados usando a técnica proposta por
MARTZ \& WALLER (1982). O modelo selecionado representa a opinião do especialista baseada em experiência passada e em evidências numéricas disponíveis até a data do estudo.

\subsubsection{Revisão da Confiabilidade dos Componentes do Produto com os Dados de Teste (Distribuição a Posteriori)}

Suponha que $n_{\mathrm{i}}$ unidades são testadas e que $s_{\mathrm{i}}$ sobreviventes foram observados, então a f.d.p. a posteriori para $R_{i}$ é dada por:

$$
h_{i}\left(R_{i} / s_{\mathrm{i}}\right)=\frac{\Gamma\left(n_{\mathrm{i}}^{\prime}+2\right)}{\Gamma\left(s_{\mathrm{i}}^{\prime}+1\right) \Gamma\left(n_{\mathrm{i}}^{\prime}-s_{i}^{\prime}+1\right)} R_{i}^{s_{i}^{\prime}}\left(1-R_{i}\right)^{n_{\mathrm{i}}^{\prime}-s_{\mathrm{i}}^{\prime}}
$$


Isto é, a distribuição da confiabilidade modelo Beta de parâmetros $n_{\mathrm{i}}^{\prime}=n_{\mathrm{i} 0}+n_{\mathrm{i}}$ e após incorporar os dados de teste é um $s_{\mathrm{i}}^{\prime}=s_{\mathrm{i} 0}+s_{\mathrm{i}}$, ou seja:

$$
R_{i} \sim \beta\left(s_{\mathrm{i}}^{\prime}+1, n_{\mathrm{i}}^{\prime}-s_{\mathrm{i}}^{\prime}+1\right)
$$

\subsubsection{Distribuição de Produtos a Posteriori}

É possível derivar a distribuição a posteriori da confiabilidade do produto a partir de dados de teste dos componentes.

Para um sistema em série, composto de $\mathrm{k}$ componentes, a confiabilidade $(\boldsymbol{R})$ é dada

\section{Modelo probabilístico}

A distribuição exata da confiabilidade $\boldsymbol{R}$ é a distribuição do produto de $\mathrm{k}$ variáveis aleatórias Beta independentes, onde a i-ésima variável tem a distribuição $a$ posteriori Beta dada pela equação 3.3. Neste trabalho, para obter o modelo probabilístico pelo produto das confiabilidades dos componentes. Isto é:

$$
R=\prod_{i=1}^{i=k} R_{i}
$$

da confiabilidade de produto, foi utilizado uma aproximação da distribuição exata, sugerida por THOMPSON \& HAYNES (1980). Esta distribuição é uma Beta de parâmetros $\boldsymbol{a}$ e $\boldsymbol{b}$ e será notada como $h_{a}(R)$, ou seja,

$$
R / s \sim \beta(a, b): h_{a}(R)
$$

com:

$$
\begin{gathered}
a=\frac{M^{2}(1-M)-V M}{V} \\
b=\frac{M(1-M)^{2}-V(1-M)}{V}
\end{gathered}
$$

parâmetros tais que a Beta tenha média $\mathrm{M}$ e variância $\mathrm{V}$, que são a média e a variância do produto de Betas, ou seja:

$$
\begin{gathered}
M=\prod_{i=1}^{i=k} \frac{\left(s_{\mathrm{i} 0}+s_{\mathrm{i}}+1\right)}{\left(n_{\mathrm{i} 0}+n_{\mathrm{i}}+2\right)} \\
V=\prod_{i=1}^{i=k}\left\{\frac{\left(s_{\mathrm{i} 0}+s_{\mathrm{i}}+1\right)\left(s_{\mathrm{i} 0}+s_{\mathrm{i}}+2\right)}{\left(n_{\mathrm{i} 0}+n_{\mathrm{i}}+2\right)\left(n_{\mathrm{i} 0}+n_{\mathrm{i}}+3\right)}\right\}-M^{2}
\end{gathered}
$$

É necessário examinar a qualidade desta aproximação Beta, antes de obter as estimativas da confiabilidade. 


\section{Estimadores de Bayes para a confiabilidade do produto}

Para uma função de perda erro quadrático, a estimativa bayesiana pontual é a média da distribuição a posteriori de $\boldsymbol{R}$ :

$$
E(R / s)=\prod_{\mathrm{i}=1}^{\mathrm{i}=k} \frac{\left(s_{\mathrm{i} 0}+s_{\mathrm{i}}+1\right)}{\left(n_{\mathrm{i} 0}+n_{\mathrm{i}}+2\right)}=\mathrm{M}
$$

Uma estimativa bayesiana por intervalo de $90 \%$ para $\mathrm{R}$ é dada pelos quantis 0,05 e 0,95 de sua distribuição a posteriori.

\subsection{Resultados Numéricos para o IBM 8240}

O exemplo apresentado aqui, apesar dos números serem apenas ilustrativos, é uma aplicação da metodologia proposta, que foi realizada em dois momentos do processo de desenvolvimento do produto. Os primeiros resultados foram obtidos na fase DVT. As estimativas obtidas foram atualizadas após o MVT. É importante enfatizar que a metodologia apresentada foi desenvolvida de acordo com o ambiente físico da informação disponível para o produto.

O conjunto de componentes de um produto IBM é definido internamente como FRUs (Field Replaceable Units). Um FRU é o componente mínimo de uma máquina plausível de substituição em caso de falha, para restaurar sua operação.

\subsubsection{Distribuição de FRU a priori}

Participaram deste trabalho profissionais dos departamentos de Projeto, Fabricação e de Garantia de Qualidade de Produto, sob o suporte da analista (autora do trabalho). A informação utilizada para definir o modelo $a$ priori foi baseada nos valores de previsão de confiabilidade do produto, bem como na opinião dos especialistas, fundamentada em seu know how e em dados de desempenho em campo de produtos IBM anteriores. Os dados de previsão foram obtidos utilizando metodologia convencionada e bancos de dados da própria empresa.

Uma das convenções foi que os cálculos de previsão de confiabilidade baseados nestas fontes tem a característica de ser valores conservadores (a favor da segurança). Por exemplo, dados de campo do produto IBM 5394 revelaram que a taxa de falhas real dos componentes eletrônicos é
$40 \%$ do valor predito, dado equivalente a um tempo médio entre falhas (MTBF) 2,5 vezes maior. A opinião dos engenheiros envolvidos no projeto é de que o valor predito representaria um limite inferior de desempenho do produto no campo. E, de acordo com os dados de campo dos produtos IBM, a expectativa para o produto em estudo é de que sua confiabilidade seja melhor em relação ao valor predito.

Partindo da convenção anterior, a equipe de desenvolvimento achou mais confortável adotar uma atitude ainda "pessimista" (a favor da segurança). Em lugar de esperar uma "melhoria" de 2,5 vezes no valor predito do MTBF do produto, supõe-se apenas uma "melhoria" de 1,4 vezes. É neste contexto que o exemplo numérico a seguir foi desenvolvido. 


\section{Esquema do Estudo da Confiabilidade do Produto IBM 8240}

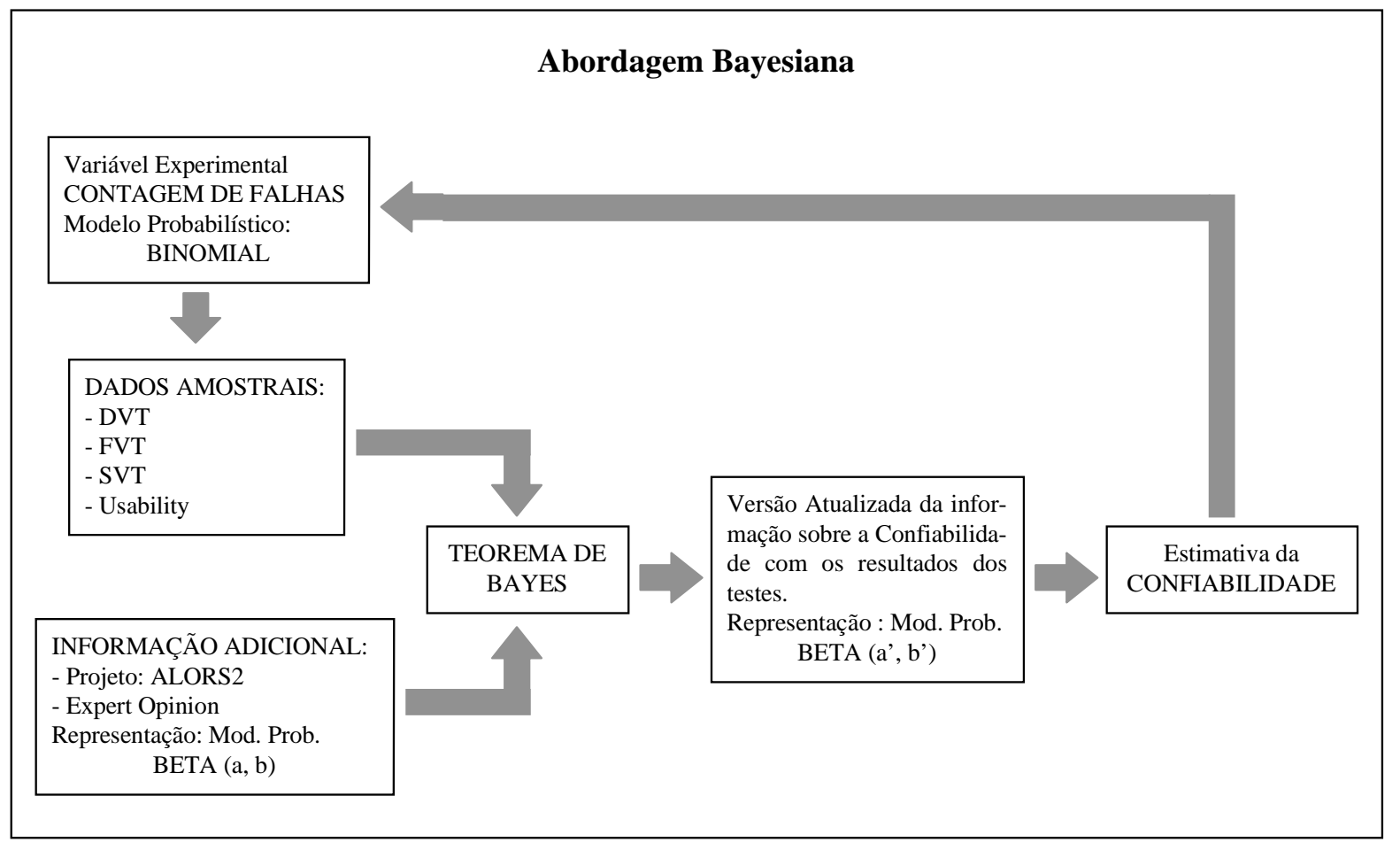

Figura 3.1. Esquema do modelo de estudo de confiabilidade aplicado ao produto IBM 8240

Para determinar os parâmetros $n_{\mathrm{i} 0}$ e $s_{\mathrm{i} 0}$ do modelo a priori, utilizando o método de Martz e Waller, é necessário definir dois valores da confiabilidade do item: o valor esperado e o $95^{\circ}$ percentil (ou o $5^{\circ}$ percentil).

Pelo exposto acima, para este problema foi conveniente definir o $5^{-}$percentil, pois se supôs que o banco de dados usado para a previsão de confiabilidade era conservador. Assim, o $5^{-}$percentil significa que a chance de valores de confiabilidade serem piores que este valor é de 5\%. E, o valor esperado será aquele que represente uma expectativa mais realista.

Assumindo como modelo de vida a distribuição exponencial, o que é coerente supor para produtos eletrônicos, fica imediato o cálculo da confiabilidade dos
FRUs considerando-se que sua operação é de 24 horas por dia. Suponha que a previsão para o equipamento, era um MTBF equivalente a 4,26 anos. Então, esta será a referência para definir o $5^{\circ}$ percentil da distribuição a priori das confiabilidades dos FRUs.

Agora, suponha que o MTBF esperado pelos especialistas foi convencionado ser de 6 anos, valor que representa um fator de 1,4 vezes melhor que o calculado mediante metodologia de previsão, então está estabelecido o critério que servirá de base para o valor esperado da confiabilidade. Observe que dita "melhoria" equivale a estabelecer que a taxa de falhas do produto será aproximadamente $71 \%$ do valor predito. Veja a tabela 3.2. 
Tabela 3.2. Taxas de falhas obtidas a partir de um MTBF de 4,26 e 6 anos para o produto.

\begin{tabular}{|l|c|c|}
\hline FRU & $\begin{array}{c}\text { Taxa de Falhas predita } \\
(\boldsymbol{\%} / \mathbf{K P O H})=\mathbf{1 0}^{-\mathbf{5}}\end{array}$ & $\begin{array}{c}\text { Taxa de falhas esperada } \\
(\boldsymbol{\%} / \mathbf{K P O H})=\mathbf{1 0}^{-5}\end{array}$ \\
\hline A & 1,0431 & 0,7412 \\
\hline $\mathrm{B}$ & 0,4655 & 0,3307 \\
\hline $\mathrm{C}$ & 0,7579 & 0,5386 \\
\hline $\mathrm{D}$ & 0,1795 & 0,1275 \\
\hline E & 0,2315 & 0,1646 \\
\hline Produto & 2,6776 & 1,9026 \\
\hline
\end{tabular}

Apresenta-se aqui um exercício considerando um tempo de missão igual a cinco anos (equivalente a 43.800 horas), e, como se está considerando uma taxa de falhas constante, é suficiente estabelecer as taxas de falhas, para se chegar nos valores das confiabilidades requeridas. A tabela abaixo resume a informação a priori discriminada por FRU:

Tabela 3.3. Dados a priori por FRU

\begin{tabular}{|l|c|c|c|c|}
\hline FRU & $\boldsymbol{R}_{1, \mathbf{i}}$ & $\boldsymbol{R}_{\mathbf{3 , \mathbf { i }}}$ & $\boldsymbol{n}_{\mathbf{i} \mathbf{0}}$ & $\boldsymbol{s}_{\mathbf{i} \mathbf{0}}$ \\
\hline $\mathrm{A}$ & 0.72 & 0.63 & 72 & 52 \\
\hline $\mathrm{B}$ & 0.87 & 0.82 & 137 & 119 \\
\hline $\mathrm{C}$ & 0.79 & 0.72 & 100 & 79 \\
\hline $\mathrm{D}$ & 0.94 & 0.92 & 427 & 401 \\
\hline $\mathrm{E}$ & 0.93 & 0.90 & 224 & 208 \\
\hline
\end{tabular}

$\boldsymbol{n}_{\mathbf{i 0}}$ e $\boldsymbol{s}_{\mathbf{i 0}}$ são os parâmetros da distribuição Beta a priori do i-ésimo FRU. $\boldsymbol{R}_{1, \mathbf{i}}$ representa a média da distribuição a priori da confiabilidade do FRU $\left(E\left(R_{i}\right)\right)$, onde $R_{1=} e^{-\lambda_{1} t}=e^{-0,438 \lambda_{1}}$, com $\lambda_{1}$ a taxa de falhas esperada; $\boldsymbol{R}_{3, \mathbf{i}}$ representa o $5^{0}$ percentil da distribuição a priori da confiabilidade do FRU $\quad\left(\quad P\left(R<\boldsymbol{R}_{3, \mathbf{i}}\right)=0,05 \quad\right), \quad$ com $R_{3}=e^{-\lambda_{3} t}=e^{-0,438 \lambda_{3}}$, e $\lambda_{3}$ a taxa de falhas obtida por métodos de previsão. 


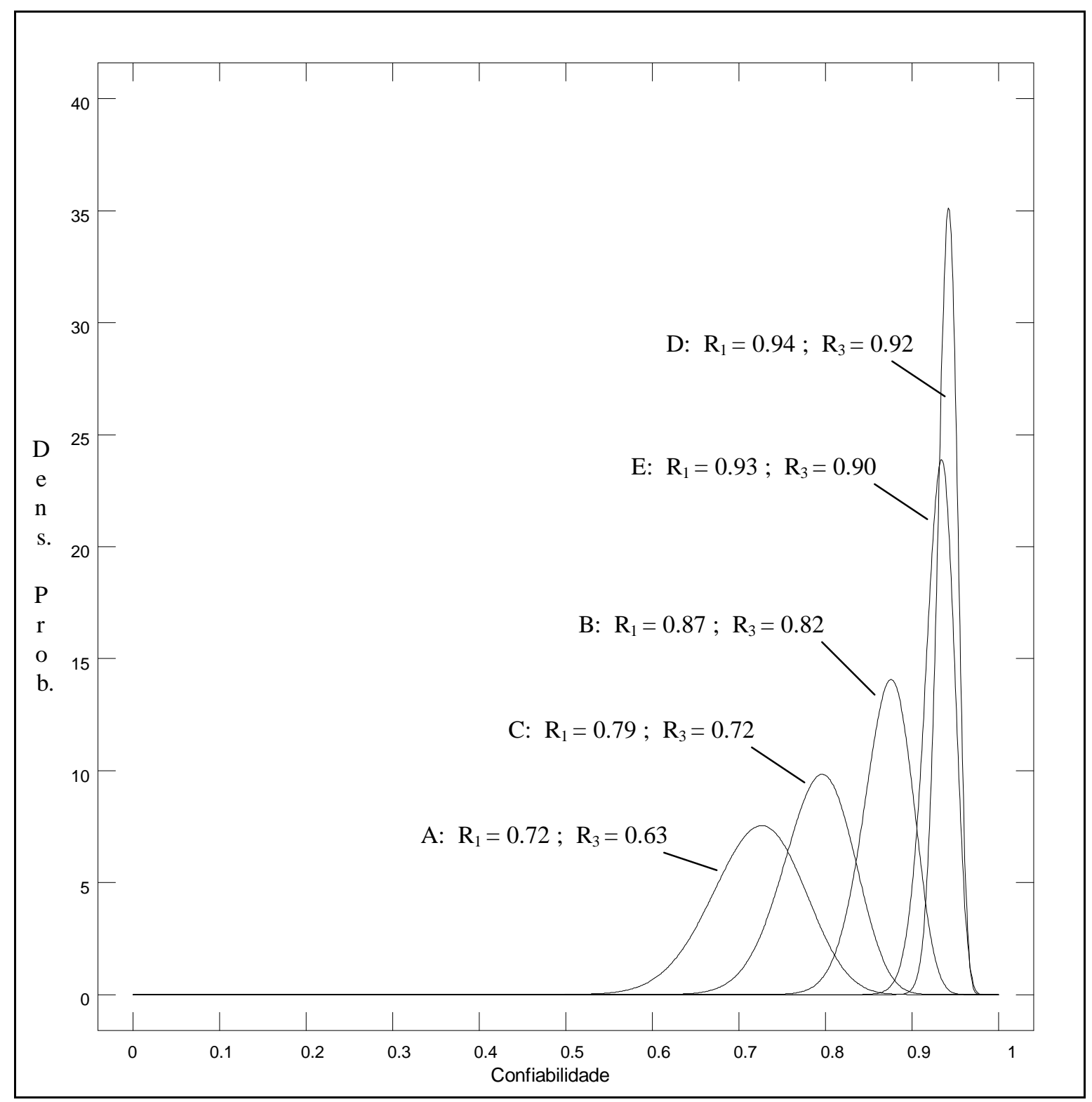

Figura 3.2. Representação probabilística da informação a priori do DVT, sobre as confiabilidades dos componentes do produto.

Das operações matemáticas resumidas na Tabela 3.3, se deduz a seguinte relação: fixado $R_{3}$, o valor da confiabilidade esperada pode ser expressa como $R_{1}=\left(R_{3}\right)^{0,71}$. Observe que, $R_{1}=e^{-0,438 \lambda_{1}}=e^{-0,438 \times 0,71 \lambda_{3}}=$ $=\left(e^{-0,438 \lambda_{3}}\right)^{0,71}=R_{3}{ }^{0,71}$.

MARTZ \& WALLER (1982) fornecem tabelas que podem ser usadas para achar os parâmetros da Beta, porém se alguma combinação não for encontrada, os mesmos podem ser calculados recorrendo-se a um método iterativo. Neste trabalho, para avaliar os parâmetros, utilizou-se os recursos do Microsoft EXCEL e, para os gráficos, o Statgraphics. As respectivas distribuições Beta estão representadas na figura 3.2.

Dispondo de dados de ensaios, será possível revisar a distribuição a priori determinada para cada FRU, obtendo-se assim suas correspondentes distribuições a posteriori. 


\subsubsection{Distribuição de FRU a Posteriori}

Considerou-se amostras do mesmo nível de hardware as máquinas dedicadas às atividades de DVT, FVT, SVT e Usability. Os resultados observados durante o período pré-definido de ensaio, estão sumarizados na
Tabela 3.4, onde $n_{\mathrm{i}}$ representa o número total de unidades testadas, $f_{\mathrm{i}}$ é o número observado de unidades falhas e $s_{\mathrm{i}}$ o número de unidades com operação satisfatória, para i $=\mathrm{A}, \mathrm{B}, \mathrm{C}, \mathrm{D}, \mathrm{E}$.

Tabela 3.4. Resultados dos testes por FRU

\begin{tabular}{|c|c|c|c|}
\hline FRU & $\boldsymbol{n}_{\mathbf{i}}$ & $\boldsymbol{f}_{\mathbf{i}}$ & $\boldsymbol{s}_{\mathbf{i}}$ \\
\hline $\mathrm{A}$ & 27 & 0 & 27 \\
\hline $\mathrm{B}$ & 27 & 3 & 24 \\
\hline $\mathrm{C}$ & 145 & 4 & 141 \\
\hline $\mathrm{D}$ & 27 & 0 & 27 \\
\hline $\mathrm{E}$ & 19 & 1 & 18 \\
\hline
\end{tabular}

Tabela 3.5. Dados a posteriori por FRU

\begin{tabular}{|c|c|c|c|c|c|}
\hline FRU & $n_{i}^{\prime}$ & $s_{i}^{\prime}$ & $\boldsymbol{a}_{\mathbf{i}}^{\prime}$ & $\boldsymbol{b}_{\mathbf{i}}^{\prime}$ & $\boldsymbol{R}_{\mathbf{i}}$ \\
\hline $\mathrm{A}$ & 99 & 79 & 80 & 21 & 0.79 \\
\hline $\mathrm{B}$ & 164 & 143 & 144 & 22 & 0.87 \\
\hline $\mathrm{C}$ & 245 & 220 & 221 & 26 & 0.89 \\
\hline $\mathrm{D}$ & 454 & 428 & 429 & 27 & 0.94 \\
\hline $\mathrm{E}$ & 243 & 226 & 227 & 18 & 0.93 \\
\hline
\end{tabular}

Na Tabela 3.5, $a_{\mathrm{i}}^{\prime}$ e $b_{i}^{\prime}$ são parâmetros da distribuição Beta de FRU a posteriori após o DVT do produto. $R_{i}$ é a média da distribuição a posteriori da confiabilidade do FRU.
Para os cálculos utilizam-se as seguintes expressões:

$$
\begin{aligned}
& n_{i}^{\prime}=n_{i 0}+n_{i} ; s_{\mathrm{i}}^{\prime}=s_{i 0}+s_{i} \\
& a_{\mathrm{i}}^{\prime}=s_{\mathrm{i}}^{\prime}+1 ; b_{i}^{\prime}=n_{i}^{\prime}-s_{i}^{\prime}+1 .
\end{aligned}
$$

\subsubsection{Resultados de Produto}

Aplicando as equações da seção 3.4.3, obtém-se que os parâmetros da distribuição Beta assumem os seguintes valores: $\mathrm{a}^{(1)}=$ $104 \mathrm{e}^{(1)}=90$. Assim, $\beta\left(\mathrm{a}^{(1)}, \mathrm{b}^{(1)}\right)$ é $\mathrm{a}$ distribuição que representa a confiabilidade do produto após o DVT.

Uma estimativa pontual para a confiabilidade do produto é dada pela média desta distribuição, cujo valor é de 0,54 (MTBF equivalente: $1 / \lambda=-\mathrm{t} / \ln \mathrm{R}(\mathrm{t})=-5 / \ln 0,54=$ 8 anos).

Da distribuição $\beta\left(a^{(1)}, b^{(1)}\right)$, que representa a confiabilidade do 8240, obtém-se o intervalo probabilístico de $90 \%$, o qual é dado pelo $5^{\mathrm{o}}$ percentil $(0,48)$ e pelo $95^{\circ}$ percentil $(0,59)$. Em termos de MTBF, isto é equivalente a 6,8 anos e 9,5 anos respectivamente. 


\subsubsection{Avaliação da Qualidade da Distribuição Aproximada}

Para que as afirmações da seção anterior sejam válidas é necessário avaliar se a aproximação utilizada não é distante da distribuição exata da confiabilidade do produto. Para isto obteve-se uma estimativa da densidade exata mediante o método de simulação de Monte Carlo, gerando 1000 observações da variável $R=\prod_{i=1}^{i=k} R_{i}$, onde $k=5$ e cada $R_{i} \sim \beta\left(s_{\mathrm{i}}^{\prime}+1, n_{\mathrm{i}}^{\prime}-s_{\mathrm{i}}^{\prime}+1\right)$.

A qualidade da aproximação pode ser avaliada comparando os gráficos das distribuições acumuladas de ambas as densidades ou também comparando alguns quantis das mesmas:

Tabela 3.6. Avaliação da qualidade da aproximação

\begin{tabular}{llll}
\hline Distribuição & Percentil 5 & Média & Percentil 95 \\
\hline Exata simulada & 0.479 & 0.538 & 0.592 \\
Aproximada & 0.48 & 0.54 & 0.59 \\
\hline
\end{tabular}

Para a simulação foi utilizado o software MINITAB, versão para Windows. Dos resultados mostrados na tabela pode-se afirmar que a qualidade da aproximação é muito boa e portanto as inferências são válidas.

\subsubsection{Revisão dos Cálculos Após o MVT}

Durante esta etapa do processo de desenvolvimento, foram obtidos novos resultados de ensaios realizados com amostras de lotes de produção em volume, os quais foram utilizados para atualizar o conhecimento, que foi obtido após o DVT, a respeito da confiabilidade do equipamento em estudo.
A informação obtida na etapa anterior viria a representar o conhecimento a priori para este novo conjunto de dados. Na Tabela 3.5 apresentam-se os dados obtidos após o DVT para determinação dos parâmetros da distribuição a priori, e na Tabela 3.7 os resultados amostrais obtidos durante o MVT.

\section{Tabela 3.7. Informação a priori para os resultados amostrais obtidos durante o MVT}

\begin{tabular}{|l|c|c|}
\hline FRU & $n_{i}^{\prime}$ & $s_{i}^{\prime}$ \\
\hline $\mathbf{A}$ & 99 & 79 \\
\hline $\mathbf{B}$ & 164 & 143 \\
\hline $\mathbf{C}$ & 245 & 220 \\
\hline $\mathbf{D}$ & 454 & 428 \\
\hline $\mathbf{E}$ & 243 & 226 \\
\hline
\end{tabular}


Tabela 3.8. Dados de ensaios realizados durante o MVT para cada FRU

\begin{tabular}{|l|c|c|c|}
\hline FRU & $n_{\mathrm{i}}$ & $f_{\mathrm{i}}$ & $s_{\mathrm{i}}$ \\
\hline $\mathbf{A}$ & 15 & 0 & 15 \\
\hline $\mathbf{B}$ & 15 & 0 & 15 \\
\hline $\mathbf{C}$ & 33 & 0 & 33 \\
\hline $\mathbf{D}$ & 15 & 0 & 15 \\
\hline $\mathbf{E}$ & 15 & 0 & 15 \\
\hline
\end{tabular}

Onde:

$n_{\mathrm{i}}$ : Número total testado deste FRU.

$f_{\mathrm{i}}$ : Número total de FRU's que falharam durante o período do ensaio estendido.

$s_{\mathrm{i}}$ : Número total de FRU's sobreviventes após a conclusão do ensaio.

Tabela 3.9. Parâmetros da distribuição a posteriori do MVT para cada FRU com suas correspondentes médias

\begin{tabular}{|l|c|c|c|c|c|}
\hline FRU & $\boldsymbol{n}_{i}^{\prime \prime}$ & $\boldsymbol{s}_{i}^{\prime \prime}$ & $\boldsymbol{a}_{\boldsymbol{i}}^{\prime \prime}$ & $\boldsymbol{b}_{\boldsymbol{i}}^{\prime \prime}$ & Média \\
\hline $\mathbf{A}$ & 114 & 94 & 95 & 21 & 0.82 \\
\hline B & 179 & 158 & 159 & 22 & 0.88 \\
\hline $\mathbf{C}$ & 278 & 253 & 254 & 26 & 0.91 \\
\hline $\mathrm{D}$ & 469 & 443 & 444 & 27 & 0.94 \\
\hline $\mathbf{E}$ & 258 & 241 & 242 & 18 & 0.93 \\
\hline
\end{tabular}

Onde $a^{\prime \prime}$ e $b^{\prime \prime}$ são parâmetros da distribuição Beta a posteriori do MVT de FRU

$$
\begin{gathered}
a_{\mathrm{i}}^{\prime \prime}=s_{i}^{\prime \prime}+1 \\
b_{i}^{\prime \prime}=n_{i}^{\prime \prime}-s_{i}^{\prime \prime}+1
\end{gathered}
$$

obtidos de acordo com:

\subsubsection{Atualização da Confiabilidade do Equipamento}

A partir dos resultados obtidos por FRU (ver Tabela 3.9), pode-se calcular a confiabilidade do 8240. Para isso, é necessário obter os parâmetros da distribuição a posteriori de produto, os quais são:

$$
\begin{gathered}
a^{(2)}=124 \\
b^{(2)}=93
\end{gathered}
$$

A estimativa da média desta distribuição Beta $\left(\mathrm{a}^{(2)}, \mathrm{b}^{(2)}\right)$ é 0,57 , o que dá um MTBF equivalente a 9 anos.

O verdadeiro valor da confiabilidade do produto encontra-se com 0,90 de probabilidade no intervalo $(0,52 ; 0,63)$; este resultado, expresso em termos de MTBF, é equivalente a 7,6 e 10,8 anos respectivamente.

\section{Conclusões}

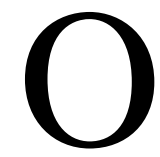

procedimento utilizado neste trabalho tem a principal vantagem de aproveitar toda informação disponível para o estudo, seja objetiva e/ou subjetiva. Não sendo a quantidade de ensaios a única e exclusiva fonte de informação, os custos de desenvolvimento foram grandemente beneficiados. Possibilitou uma 
melhor compreensão das falhas observadas no produto, pois os testes foram realizados em condições normais de operação. A qualidade das inferências obtidas foi superior em relação à proposta baseada na estatística clássica. A metodologia proposta possibilita adaptar as ferramentas estatísticas ao cenário de desenvolvimento de maneira simples e econômica.
Este trabalho não é exclusividade da indústria eletrônica, há um campo potencial de aplicação dos métodos bayesianos na área fabril de equipamentos mecânicos, em que o conhecimento do especialista tem um papel muito importante. Também, pode-se afirmar que problemas, de difícil tratamento matemático na área de confiabilidade de software, podem ser facilmente resolvidos por técnicas Bayesianas.

\section{Referências Bibliográficas:}

COLE, P.V.Z.: “A bayesian reliability assessment of complex systems for binomial sampling". IEEE Transactions on Reliability, v.24, n.2, June 1975, pp 114-117.

CROOK, D.L.: "Evolution of VLSI reliability engineering". Quality and Reliability Engineering International, v.7, 1991, pp 221-233.

GARVIN, D.A.: Gerenciando a qualidade. Brasil, Qualitymark Editora, 1992.

HART, L.: "Reliability of modified designs: a Bayes analysis of an accelerated test of electronic assemblies". IEEE Transactions on Reliability, v.39, n.2, June 1990, pp 140-144.

MARTZ, H.F. \& WALLER, R.A.: "Bayesian reliability analysis of complex series/parallel systems of binomial subsystems and components". Technometrics, v.32, n.4, November 1990, pp 407-416.
: Bayesian reliability analysis. USA, John Wiley \& Sons, 1982.

MARTZ, H.F.; WALLER, R.A. \& FICKAS, E.T.: "Bayesian reliability analysis of series systems of binomial subsystems and components". Technometrics, v.30, n.2, May 1988, pp 143-154.

MASTRAN, D.V.: "A bayesian approach for assessing the reliability of air force re-entry systems". Proc. Ann. R\&M Conf., 1968, pp 380383.

MEEKER, W.Q. \& HAMADA, M.: "Statistical tools for the rapid development $\&$ evaluation of high reliability products". IEEE Transactions on Reliability, v.44, n.2, June 1995, pp 187-198.

THOMPSON, F.A. \& HAYNES, R.D.: "On the reliability, availability and Bayes confidence intervals for multicomponents systems". Naval Research Logistics Quarterly, v.27, 1980, pp 345-358.

\section{A BAYESIAN METHODOLOGY FOR RELIABILITY STUDIES IN THE DESIGN PHASE: APPLICATION TO AN ELECTRONIC PRODUCT}

\section{Abstract}

Nowadays industries have to place their products in a highly competitive market, and there have been increasing pressures on efficiency test programs and product development cycles. In this context, the classical statistical methods (based on sampling theory), which are used to analyze product reliability, are negatively affected, while the bayesian methods are favorably regarded, and their use is becoming a necessary alternative.

In order to evaluate the performance of an item with a very low failure rate, the classical statistical techniques require a large sample size and a long test time. This is particularly true 
when the product technology limits the acceleration factor, as with electronic products, for example.

The methodology proposed in this paper combines test results, which are routinely performed during the product development cycle, with additional relevant information that is useful in the assessment of its reliability.

In order to illustrate the methodology, it was applied to an electronic equipment, assessing its reliability during the design phase. The computations were performed considering component reliabilities, attribute test data, and also judgement of the product development team.

Key words: reliability, bayesian statistics, quality, failure rate, expert opinion. 